\title{
Astrometric search for a planet around VB 10
}

\author{
P. F. Lazorenko ${ }^{1}$, J. Sahlmann², D. Ségransan ${ }^{2}$, P. Figueira ${ }^{2,5}$, C. Lovis ${ }^{2}$, E. Martin ${ }^{3}$, M. Mayor ${ }^{2}$, F. Pepe ${ }^{2}$, D. Queloz ${ }^{2}$, \\ F. Rodler ${ }^{4}$, N. Santos ${ }^{5,6}$, and S. Udry ${ }^{2}$
}

1 Main Astronomical Observatory, National Academy of Sciences of the Ukraine, Zabolotnogo 27, 03680 Kyiv, Ukraine e-mail: laz@mao.kiev.ua

2 Observatoire de Genève, Université de Genève, 51 Chemin des Maillettes, 1290 Sauverny, Switzerland

3 INTA-CSIC Centro de Astrobiología, 28850 Torrejón de Ardoz, Madrid, Spain

4 Instituto de Astrofísica de Canarias, C/ Vía Láctea s/n, 38200 La Laguna (Tenerife), Spain

5 Centro de Astrofísica, Universidade do Porto, Rua das Estrelas, 4150-762 Porto, Portugal

6 Departamento de Física e Astronomia, Faculdade de Ciências, Universidade do Porto, Portugal

Received 13 July 2010 / Accepted 5 November 2010

ABSTRACT

\begin{abstract}
We observed VB 10 in August and September 2009 using the FORS2 camera of the VLT with the aim of measuring its astrometric motion and of probing for the presence of the announced planet VB 10b. We used the published STEPS astrometric positions of VB 10 over a timespan of 9 years, which allowed us to compare the expected motion of VB 10 due to parallax and proper motion with the observed motion and to compute precise deviations. The single-epoch precisions of our observations are about 0.1 mas, and the data showed no significant residual trend, while the presence of the planet should have induced an apparent proper motion greater

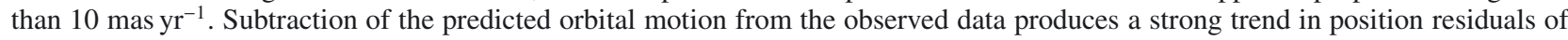
VB 10. We estimated the probability that this trend is caused by random noise. After taking all the uncertainties into account and using Monte-Carlo resampling of the data, we are able to reject the existence of VB $10 \mathrm{~b}$ with the announced mass of $6.4 M_{\mathrm{J}}$ with a false alarm probability of only $5 \times 10^{-4}$. A $3.2 M_{\mathrm{J}}$ planet is also rejected with a false alarm probability of 0.023 .
\end{abstract}

Key words. astrometry - techniques: high angular resolution - planetary systems - stars: individual: VB 10

\section{Introduction}

The detection of a planetary-mass companion to the nearby M8 ultracool dwarf VB 10 (GJ 752B) was announced by Pravdo \& Shaklan (2009). Using ground-based astrometric observations over nine years with the STEPS camera of the $5 \mathrm{~m}$ Palomar telescope, the authors derived a full astrometric solution for the system that suggests a planetary companion with a 271 day orbital period and $6.4 M_{\mathrm{J}}$ mass. Even though the VB $10 \mathrm{~b}$ astrometric signature is large with a peak-to-peak value of 8 mas, the detection is debatable because of imperfect orbital sampling and the comparably low astrometric accuracy of the individual measurements.

Zapatero Osorio et al. (2009) presented high-resolution nearinfrared observations of VB 10 obtained between 2001 and 2008 with the NIRSPEC instrument on the Keck II telescope. They emphasize the necessity for a better sampling of the orbital phase to constrain the orbital parameters and the individual masses of the system precisely. Bean et al. (2010) used the near-infrared CRIRES spectrograph and did not detect the planet's signature in radial-velocity (RV). Anglada-Escudé et al. (2010) performed a joint analysis of precise RV observations over 175 days with MIKE/Magellan and ESPaDOnS/CFHT spectrographs, RV measurements by Zapatero Osorio et al. (2009), and astrometric data by Pravdo \& Shaklan (2009). They show that the observed astrometric motion does not come from an unseen companion.

^ Based on observations made with ESO telescopes at the La Silla Paranal Observatory under programme ID 283.C-5024.
We present results of the astrometric search for the planet using the FORS2 camera of the VLT, the only instrument that can achieve astrometric precision of individual measurements of 0.05-0.1 mas (Lazorenko et al. 2009). Despite the short observation period of two months, we obtained good enough data to verify the hypothesis that VB 10b exists.

\section{Observations}

Observations were made with the FORS2/VLT camera (Appenzeller et al. 1998) in imaging mode during five nights from 2 August to 25 September 2010. The images cover a $4.2 \times 4.2^{\prime}$ field of view with a pixel (px) scale of $\sim 0.1^{\prime \prime} / \mathrm{px}$. For each night, we obtained 21 to 72 frames of $7 \mathrm{~s}$ exposures with the $R_{\text {special }}$ filter. Seeing varied from $0.55^{\prime \prime}$ to $0.9^{\prime \prime}$. To reduce differential chromatic refraction (DCR) of the atmosphere, we observed near meridian.

Because the observed motion of VB 10 in the sky is a long arc (over 2 px), we had to know the precise pixel scale to convert pixel to arcsec units without loss of accuracy. From the CMC14 and NOMAD catalogue positions of stars in our reference frame, we derived acceptably precise scales of $0.12538 \pm 0.00007^{\prime \prime} / \mathrm{px}$ and $0.12567 \pm 0.00005^{\prime \prime} / \mathrm{px}$ in RA and Decl, respectively.

\section{Data reduction and analysis}

Our null hypothesis was that the planet VB 10b exists. To test it, we computed the residuals of our measurements compared to the position of VB 10 expected from the combination of proper, parallactic, and orbital motions. By determining the probability that 
the measured residuals are compatible with the expected mean value of zero, we could confirm or reject the null hypothesis. We also performed the corresponding computations by assuming that the planet does not exist.

In all cases, we considered that the astrometric signal $\Psi$ in VB 10 position induced by the planet is defined by the orbital elements of VB 10b given in the discovery paper by Pravdo \& Shaklan (2009). The predicted orbital motion over the measurement timespan of 17 days is 0.48 mas in RA and 0.92 mas in Dec, and for 54 days it has 1.95 mas in RA and 3.28 mas in Dec. Compared to the astrometric precision of FORS2 of about 0.1 mas per epoch, these displacements are large and should be detected in this study.

\subsection{Photocentre determination}

Raw images were flat-fielded and bias-subtracted to exclude pixel-to-pixel variations in the CCD sensitivity. To increase the number of reference stars, we measured all star images with $R=14-21$. At this faint end, the star field is very crowded. This forced us to improve our procedure of photocentre computation, which was initially developed for isolated stellar images (Lazorenko 2006). We scanned images obtained under various seeing conditions and made a detailed census of star positions at subpixel precision, of fluxes, and of the pointspread function variation across the CCD. This information was used to accurately model and subsequently subtract the contamination of background counts caused by distant stars. Wings of star profiles at distances up to $50 \mathrm{px}$ were approximated by exponential function with free parameters smoothly varying over the CCD. The photocentres $\hat{x}$ and $\hat{y}$ were computed by fitting star profiles in a $11 \times 11 \mathrm{px}$ window with a model with 12 free parameters and an auxiliary oscillating function in the central $5 \times 5$ px window (Lazorenko 2006). Computations based on the Levenberg-Marquardt numerical algorithm of the least squares fit were found to give good enough results for stars of approximately equal brightnesses at separations larger than about $10 \mathrm{px}$.

\subsection{Astrometric model}

Reductions were based on the method previously applied to FORS1/2 observations (Lazorenko et al. 2007, 2009). The method was shown to efficiently mitigate atmospheric image motion, geometric field distortion, DCR, and other effects, thus ensuring stable precision over time scales of a few days to a few years. For every star in the field, i.e. VB 10 and the reference stars, the measured photocentre positions $\hat{x}_{m}$ and $\hat{y}_{m}$ in frame $m$ at time $t$ were represented by the model

$$
\begin{gathered}
x_{0}+\Phi_{k m}^{\{x\}}(x, y)+\mu_{x} t+\pi p_{x}+\rho \tan z \sin \gamma+d \tan z_{\mathrm{L}} \sin \gamma \\
=\hat{x}_{m}-\Psi(t) .
\end{gathered}
$$

The lefthand side contains the free model parameters and the model function $\Phi_{k m}^{\{x\}}(x, y)$. The expression for $y$-data is similar but contains $\cos \gamma$ instead of $\sin \gamma$. Here, $x_{0}$ is a zero point, $k$ the mode (an arbitrary even integer, usually from 4 to 16), $\Phi_{k m}^{\{x\}}$ a polynomial in $x$ and $y$ of order $k / 2-1$, which models the sum of atmospheric image motion and geometric distortion for each frame $m$. The parameter $\mu_{x}$ is the proper motion, $\pi$ the parallax, and $p_{x}$ the parallax factor in $x$. The displacement of the star image due to DCR is modelled by a term with leading parameter $\rho$, which depends on the star's colour, and contains the zenith distance $z$ and the angle $\gamma$ between a direction to zenith and $y$-axis. The next term describes an image displacement opposite to that of DCR and introduced by the longitudinal atmospheric dispersion compensator (LADC) of the VLT (Avila et al. 1997). This displacement also depends on the star colour via the parameter $d \approx-\rho$. Both $\rho$ and $d$ are free model parameters, and their computation does not require external colour data. The LADC is automatically adjusted to the average zenith distance $z_{\mathrm{L}}$ over a given series of frames by setting a distance $b$ between its two prisms to $b \sim \tan z_{\mathrm{L}}$. Finally, $\Psi(t)$ represents the induced orbital motion of VB 10 if the planet exists. For reference stars, $\Psi(t)=0$.

Equation (1) defines a system of equations in the combined $\{x, y, t\}$-domain for which the unique solution is derived under the condition that the model parameters of reference stars are orthogonal to each basic function of $\Phi_{k}$. It is solved iteratively for all reference stars available in a circular region of radius $R_{k}$, which increases with $k$ and is centred on the target VB 10. The optimal field size $R_{k}$ is the size at which the noise from a reference field is equal to the noise from atmospheric image motion. The number of reference stars depending on $k$ varies from a few dozen to 500. Thanks to the large number of reference stars, the function $\Phi$ accurately reproduces the coordinate grid distortion introduced by image motion. Solutions for $\Phi_{\mathrm{km}}$ at each mode $k$ are then used to form equations for the target only in the time domain. The solution of this new set of equations yields the target's model parameters and position deviations from the model. The final output is the average obtained from the solutions at all modes $k$.

\subsection{Comparison of STEPS and FORS2 reference frames}

Because of different zero-points, STEPS proper motion cannot be directly applied to FORS2 astrometry. The STEPS reference frame is given by 15 bright stars. For the FORS2 reference frame, we used both these (except STEPS star Nr. 10 which is a binary) and a number of much fainter stars. The system of proper motions is defined under the condition $\sum \mu_{i} w_{i}=0$ where summation is taken over all reference stars $i$ with weights $w_{i}$ approximately proportional to their brightness (Lazorenko et al. 2009). In particular, this condition is valid for the proper motions of the STEPS stars, which due to their high brightness (they contribute to over $40 \%$ of the total light flux), are the basis of the FORS2 reference frame. One may therefore expect that $\sum \mu_{i} w_{i} / \sum w_{i}$ (the weighted mean proper motion in the system of FORS2 proper motions) taken over these stars, is nearly zero. The computed value of this sum (the difference of proper motion zero-points)

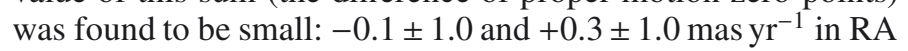
and Decl. The low precision meant that we did not apply it but included it in the error budget assuming that the uncertainty in the proper motion zero point is $\sim 1$ mas $\mathrm{yr}^{-1}$. Thus, both proper motion systems are consistent, but only on time scales of $\sim 0.1 \mathrm{yr}$.

\subsection{Short and full dataset}

The data reduction of the first epoch (2 Aug) was problematic because of incorrect telescope pointing, owing to which all stars in a 300 px wide area just below VB 10 (Fig. 1a) were imaged to another CCD chip and could not serve as a reference on this particular night. We dealt with this problem in two ways. First, we put aside all stars within this area from frames of all nights, using only stars outside of this area as reference objects. The sizes and shapes of the reference fields in this case are shown in Fig. 1a by five circular segments, each corresponding to $k$ increasing from six (small radius) to 14 (large radius). Alternatively, we 


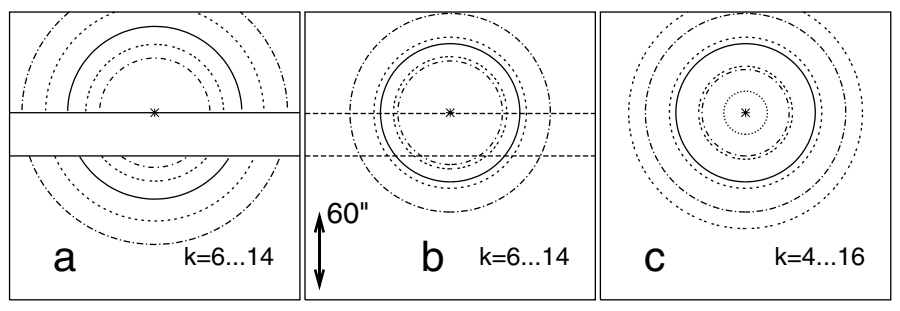

Fig. 1. Circular reference fields increasing in size with increasing modal number $k$ in the field of view of FORS2 in the case of: a) the full dataset when an area unavailable on 2 Aug. frames was cut away at all nights; b) the same but the problem area cut only at 2 Aug. frames; c) the short dataset not using 2 Aug. frames.

used circular reference areas, which for the 2 Aug. night were the same $R_{k}$ size but with the lower half vignetted (Fig. $1 \mathrm{~b}$ ). We also examined a short dataset without the 2 Aug. epoch with circular reference area (Fig. 1c). The asymmetry of the first two cases degraded the precision obtained compared to the symmetric configurations of the short dataset.

We did obtain a solution for all three cases represented in Fig. 1. For the final result, we present two solutions. One is obtained from the short dataset (case "c", MJD between 55082 and 55099 days), and the second obtained as the average of the results in cases "a" and "b". The latter solution is referred to as having been obtained from the full dataset (MJD between 55045 and 55099 days).

\subsection{Recovering $L A D C$ positions}

VB 10 is very red and differs considerably in colour from the reference stars. Therefore, the DCR displacement of this star is very large and about 30 mas in Decl and 10 mas in RA. This is taken into account by the free model parameters $\rho$ and $d$, but their adjustment requires knowledge of the LADC separation $b$.

Information on $b$ was not part of the obtained fits headers and was not accessible to us. Therefore we had to solve the inverse problem of recovering LADC separations from the observations. Because $b$ is fixed for a series of frames on a single night, we had to determine a small number of values for $b$ (one value per night). Our approach is based on the observation that incorrect values of $b$ bias the average night position residuals $\langle x\rangle$ and $\langle y\rangle$ of field stars. According to Eq. (1), this bias linearly depends on the star colour parameter $d$ during one given night. This is illustrated by Fig. 2, which shows the distribution and linear dependencies of $\langle y\rangle$ on $d$ for field stars in the case of the short dataset (the effect is greatest in $y$ ).

Because all observations were made at small hour angles within $\pm 0.7 \mathrm{~h}$, we initially assumed that LADC was always set to the separation corresponding to the meridian. By applying small corrections to these initial values of $b$, we iteratively reached a solution without dependence of $\langle y\rangle$ on colours (lower panel of Fig. 2). For these computations, we used all stars within the entire field of view of FORS2, processing them as target objects relative to their own subsets of reference stars. In spite of being based on field stars only, this procedure produced a much smaller dispersion of $\langle y\rangle$ values for VB 10 and for reference stars with extreme colours. Corrections to $b$ were small and within $\pm 4 \%$ of the initial values and were found with a precision corresponding to \pm 0.025 mas error in $\langle y\rangle$ for VB 10 . For the full dataset, precision degraded to \pm 0.068 mas because we could not use stars in the problematic 300 px area below VB 10 (Fig. 1a).

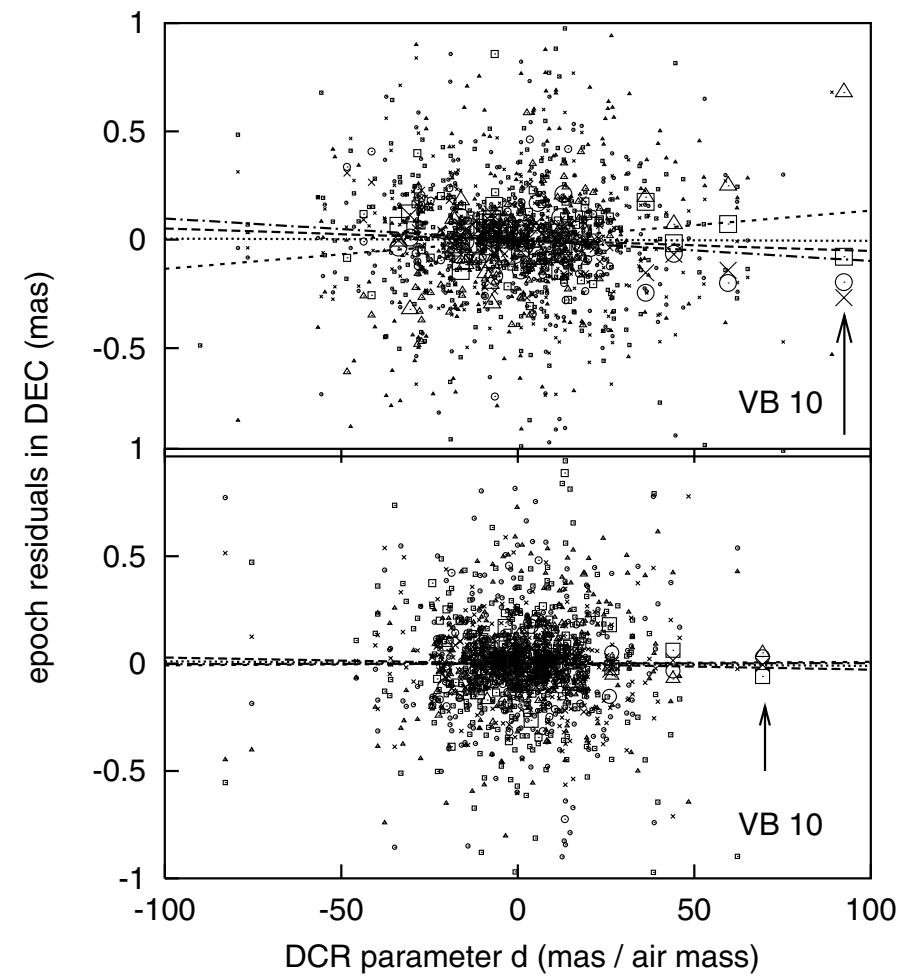

Fig. 2. Distribution of epoch position residuals $\langle y\rangle$ of field stars (dots of different type) as a function of $d$ for four September nights and linear dependences of $\langle y\rangle$ on $d$ (lines of different type) for each night. Upper panel: with initial LADC separations $b$. Lower panel: the same with recovered $b$ values. Size of dots is proportional to the star brightness.

\subsection{Treatment of colour-dependent terms}

An essential drawback of this procedure is that the recovery of $b$ introduces small colour-dependent terms $\mu^{\prime}(d)$ and $\pi^{\prime}(d)$, which are similar to proper motion and parallax. This is because the above iterations do not garantee convergence to the actual values of $b \sim \tan z_{\mathrm{L}}$. For example, the restored value of $\tan z_{\mathrm{L}}$ may differ from its actual value by a term that progressively changes in time as $\tilde{\mu} t \tan ^{-1} z_{\mathrm{L}}\langle\sin \gamma\rangle^{-1}$, where $\tilde{\mu}$ is an arbitrary constant and $\langle\sin \gamma\rangle$ is the average value of $\sin \gamma$ during the given night. As a result, the term $d \tan z_{\mathrm{L}} \sin \gamma$ in Eq. (1) generates the term $d \tilde{\mu} t \sin \gamma\langle\sin \gamma\rangle^{-1} \approx d \tilde{\mu} t$, which is linearly dependent on $d$ and which therefore can be treated as an extra image motion $\mu^{\prime}(d)=$ $\tilde{\mu} d$. The term $\mu^{\prime}(d)$ compensates for the linear change of $\tan z_{\mathrm{L}}$ in time. Similarly, we may assume that the restored values of $\tan z_{\mathrm{L}}$ contain terms proportional to $p_{x}$ and $p_{y}$. In this case, the solution of Eq. (1) for parallax should contain the compensating colour-dependent term $\pi^{\prime}(d)$.

When recovering $b$ values, we cannot control the amplitude of $\tilde{\mu}$ and of the equivalent parameter $\tilde{\pi}$ related to parallax. However, they can be detected as a proper motion and parallax dependence on colours (i.e. on $d$ ), which we model as a linear trend in proper motions of field stars and statistically correct for it.

The strong correlation between $t, p_{x}$, and $p_{y}$ does not allow us to determine $\mu^{\prime}(d)$ and $\pi^{\prime}(d)$ separately. However, this is not required because, for short times $p_{x} \sim t$, we can approximate the sum of the proper motion and parallax displacement $\mu_{x}^{\prime} t+\pi^{\prime} p_{x}$ by $\mu_{x}^{\prime} t$, where $\mu^{\prime}$ is the new effective quantity that substitutes for both $\mu^{\prime}$ and $\pi^{\prime}$. Thus by processing the short dataset, we find and use only a single colour term $\mu^{\prime}(d)$. The treatment is similar for the $y$ components. Subtracting $\mu^{\prime} t$ from the measured 


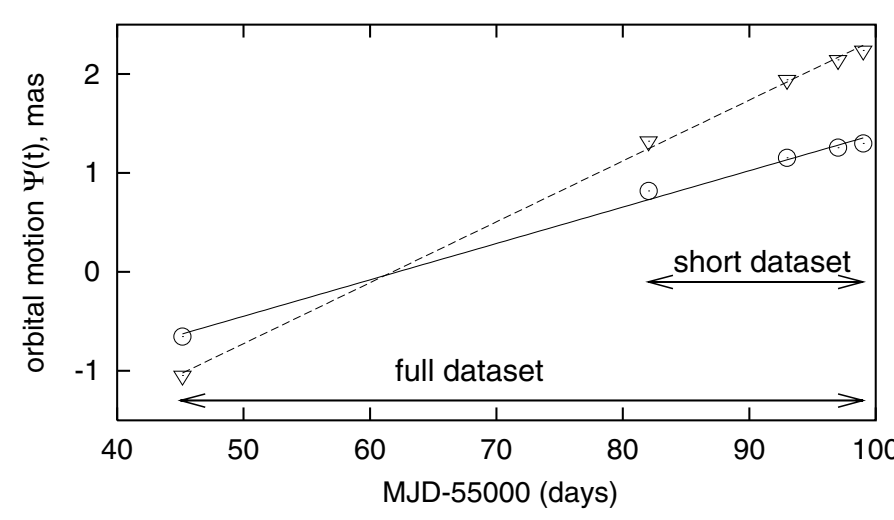

Fig. 3. Orbital motion $\Psi(t)$ of VB 10 in RA (circles) and Decl (triangles) for the full and short FORS2 datasets and the linear approximations (solid and dashed lines) of these motions.

positions thus simultaneously eliminates the colour term $\pi^{\prime}$ (see next section). The value of this correction for VB 10 in Decl is $\mu^{\prime}=29 \pm 4.5 \mathrm{mas} \mathrm{yr}^{-1}$ for the full and $\mu^{\prime}=27 \pm 2.9 \mathrm{mas} \mathrm{yr}^{-1}$ for the short dataset, respectively. In RA, the corrections are an order of magnitude smaller.

For the full dataset, the above approximation of $\mu_{x}^{\prime} t+\pi^{\prime} p_{x}$ by $\mu_{x}^{\prime} t$ is still valid for reference stars, most of which have moderate $d$ and therefore very small colour-induced parallaxes $\pi^{\prime}$. But it is not precise enough for VB 10. Because of its extreme colour term $d$, the expected $\pi^{\prime}$ value is also large and cannot be accurately eliminated by applying the correction $\mu^{\prime}(d)$ as in the case of the short dataset. Therefore, we had to treat the term $\pi^{\prime}$ of VB 10 as a free model parameter.

\subsection{Subtraction of parallax and proper motions}

Since the FORS2 observations cover a small fraction $(<20 \%)$ of the orbital period, $\Psi(t)$ can be approximated as a linear function of $t$ :

$$
\Psi_{\alpha}(t) \sim Q_{\alpha} t, \quad \Psi_{\delta}(t) \sim Q_{\delta} t,
$$

where $Q_{\alpha}=\partial \Psi_{\alpha}(t) / \partial t$ and $Q_{\delta}=\partial \Psi_{\delta}(t) / \partial t$. With the orbital elements given by Pravdo \& Shaklan (2009), we obtained $Q_{\alpha}(\mathrm{PS})=$ 13.4 and $Q_{\delta}(\mathrm{PS})=22.4$ mas yr$^{-1}$ (Fig. 3). Astrometric acceleration terms (deviations from the linearity) are smaller than 0.1 mas for the full dataset, so are negligible. For the short dataset, they are even smaller than 0.02 mas.

In addition, $\Psi(t)$ is approximately a linear function of the parallax factors $p_{x}(t)$ and $p_{y}(t)$, which themselves have an approximately linear time dependence. This causes a strong correlation between parallax, proper motion, and orbital motion, making Eq. (1) degenerated. Therefore, we subtracted parallax and proper motion from the measured positions of VB 10 . Precise values of these parameters were found based on the published STEPS astrometric measurements which cover a 9 year period. The best fit of STEPS data yielded $\mu_{\alpha} \cos (\delta)=-586.8 \pm$

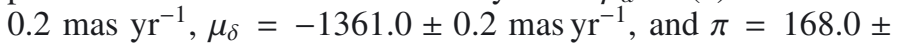
1.2 mas. These values are very close to the estimates given by Pravdo \& Shaklan (2009) and Anglada-Escudé et al. (2010).

\subsection{Position residuals of models with and without a planet}

Subtracting the contributions of parallax and proper motion from the measured positions of VB 10 resulted in simplifying Eq. (1), which for VB 10 took the form

$$
\begin{aligned}
& x_{0}+\rho \tan z \sin \gamma+d \tan z_{\mathrm{L}} \sin \gamma+\left\{\pi^{\prime} p_{x}\right\} \\
& \quad=\hat{x}_{m}-\mu_{x} t-\pi p_{x}-\mu_{x}^{\prime} t-\Phi_{k m}^{\{x\}}(x, y)-\Psi_{x}(t) \\
& y_{0}+\rho \tan z \cos \gamma+d \tan z_{\mathrm{L}} \cos \gamma+\left\{\pi^{\prime} p_{y}\right\} \\
& \quad=\hat{y}_{m}-\mu_{y} t-\pi p_{y}-\mu_{y}^{\prime} t-\Phi_{k m}^{\{y\}}(x, y)-\Psi_{y}(t)
\end{aligned}
$$

with only four free parameters $x_{0}, y_{0}, \rho$, and $d$ in the case of the short dataset. For the full dataset, it incorporates the extra parameter $\pi^{\prime}$ (the term in curly braces, see Sect. 3.6). The righthand side of Eq. (3) contains the measured coordinates $\hat{x}_{m}$ and $\hat{y}_{m}$, the inplate solutions $\Phi_{k m}^{\{x\}}(x, y)$ and $\Phi_{k m}^{\{y\}}(x, y)$ derived from the reference stars, and all other corrections.

There is a correlation between the orbital signal and the night's average angles $\langle\sin \gamma\rangle$, which are not zero and tend to increase in time. Because of this correlation, the model Eq. (1) filters out any component of the signal (e.g. $\Psi)$, which is linearly dependent on $t$ and enters the righthand side of Eq. (1). Therefore, the output position residuals only contain a part of the initial signal amplitude. However, this does not hamper our statistical analysis, which we performed after the subtraction of $\Psi(t)$ from the observed positions, thus assuming zero input signal and consequently zero output signal. In this way, the impact of correlations is minimized. If the planet does not exist but the subtraction of $\Psi(t)$ was applied, we should detect the inverse signal $-\Psi(t)$ reduced in amplitude because of the correlation between $\langle\sin \gamma\rangle$ and $\Psi(t)$. This is the case corresponding to the last row of Table 2 (Sect. 4), where the measured signal (expressed by the parameter $Q$ ) has about half its expected value $-Q(\mathrm{PS})$.

Figure 4 shows the results in terms of model deviations in Decl, where the expected signal is strongest. The single-frame precision $\sigma_{1}$ includes errors in the photocentre measurements, the reference frame noise, and the atmospheric noise. It varies from 0.4 to 0.7 mas depending on seeing. The effect of the vignetted reference field of the 2 Aug. epoch (configurations "a" and "b") is seen as a degradation of $\sigma_{1}$ to over 1 mas. At other epochs, $\sigma_{1}$ is larger compared to configuration "c" because of a larger $R_{k}$.

Clear conclusions can be drawn from the short dataset when the model (Eq. (3)) is simplest and does not require incorporation of parallax (Sect. 3.6). We considered the cases with the predicted orbital motion subtracted $(\Psi \neq 0)$ and with $\Psi=0$. The epoch average deviations $\langle x\rangle$ and $\langle y\rangle$ are very small and randomly scattered when assuming $\Psi=0$, but display a negative trend in time if $\Psi \neq 0$. Small position deviations do not correspond to a "zero" measurement. Instead, they demonstrate very precise position measurements, which track the proper motion and parallax displacement at the daily rate of 2.7 mas and 5.0 mas in RA and Dec, respectively. The motion of VB 10 over the CCD surface (Fig. 5) for the measurement timespans of 17 days is 46 mas in RA and 86 mas in Decl, and is dominated by parallax and proper motion. DCR effects induce a small-scale scatter in the measured positions of one night with an amplitude of about 2 mas. Their structure for a typical night is shown in 5-fold magnification in Fig. 5.

Similar computations were performed for the full dataset, where we had to account for the parallax correction $\pi^{\prime}(d)$ (see Sect. 3.6), so used the five parameters $x_{0}, y_{0}, \rho, d$, and $\pi^{\prime}$ for fitting of the model Eq. (3). This introduced a strong correlation between $p_{x}, p_{y}$, and $\Psi$, which reduced the amplitude of the detected signal.

Table 1 and Fig. 6 summarize the results for the epoch residuals $\langle x\rangle$ and $\langle y\rangle$. The astrometric precision is described by a nominal precision $\sigma_{\mathrm{N}}$ based on errors in photocentre determination, the reference frame noise and atmospheric noise. The term 

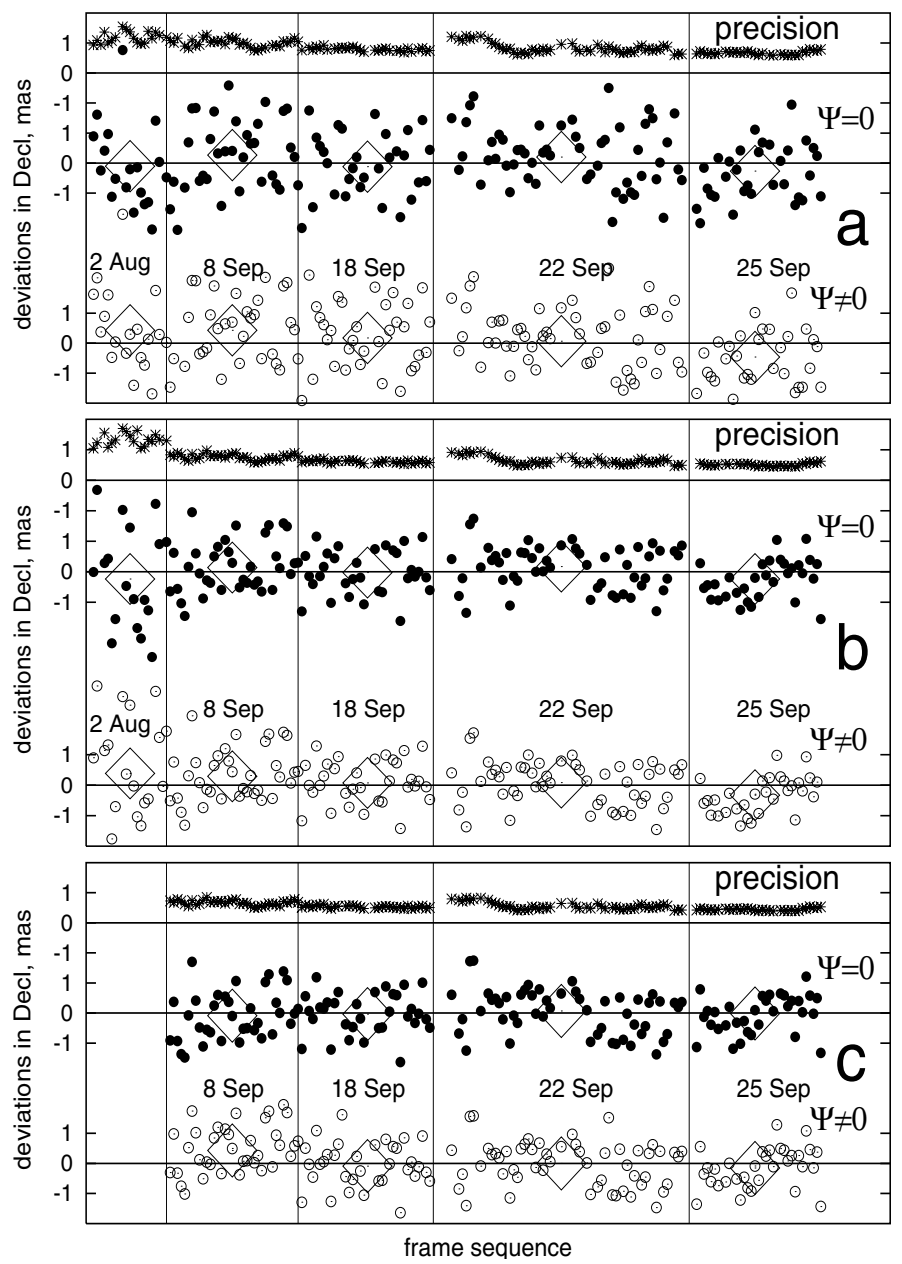

Fig. 4. Frame-to-frame deviations in Decl of VB 10 from its model motion at zero $(\Psi=0$, filled circles $)$ and non-zero planetary signal $(\Psi \neq 0$, open circles), the epoch average deviations (large diamonds), and the single-frame precisions $\sigma_{1}$ (asterisks) for the cases $\mathbf{a}$ ), b), and $\mathbf{c}$ ) of reference field configurations shown in Fig.1.

$\sigma_{\text {sum }}$ also includes error components that dominate at long time spans and come from the uncertainties in $b, \mu^{\prime}$, pixel scale, and proper motion of VB 10. For the full dataset, it also includes the uncertainty in the parallax of VB 10. The mathematical expectation of the root-mean-square of $\langle x\rangle$ and $\langle y\rangle$, derived from the least squares fit (Eq. (3)) is given by $\sigma_{\text {fit }}$.

\section{Discussion}

Because the signal $\Psi$ was subtracted in Eq. (3), the epoch deviations $\langle x\rangle$ and $\langle y\rangle$ should have an expectation value of zero and a random scatter irrespective of the model (with or without planet). A small dispersion of $\langle x\rangle$ and $\langle y\rangle$ values indicates that the tested hypothesis is correct. The data shown in Fig. 6 and Table 1 is more consistent with $\Psi=0$ (the hypothesis of no planetary companion), while incorporating a non-zero planetary signal $\Psi \neq 0$ increases the scatter of data points, which does not support the existence of the planet VB 10b. We estimated the probability $P$ that the planet exists in two ways.

The first estimate $P\left(\chi^{2}\right)$ was based on the values of $\chi^{2}=$ $\sum\left[\langle x\rangle^{2} / \sigma_{\text {sum }}^{2}+\langle y\rangle^{2} / \sigma_{\text {sum }}^{2}\right]$, where the sum is taken over epochs. The number of degrees of freedom (d.o.f.) is equal to the number of epochs minus the number of model parameters related to the time domain. There are two $\left(x_{0}, y_{0}\right)$ and three $\left(x_{0}, y_{0}, \pi^{\prime}\right)$

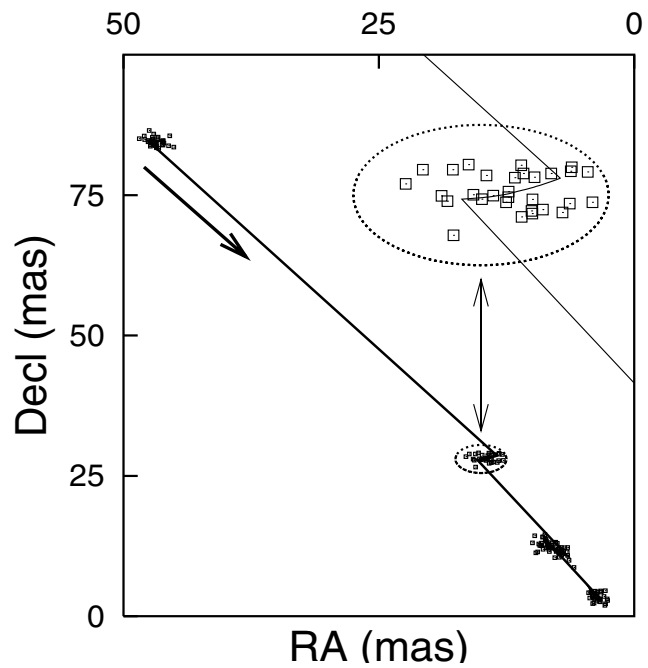

Fig. 5. Measured in-frame positions $\hat{x}-\Phi^{\{x\}}, \hat{y}-\Phi^{\{y\}}$ (squares) of VB 10, the model motion (solid line) over the CCD between 8 and 25 Sept. (short dataset) defined primarily by proper motion and parallax, and the magnified DCR signature for a single night (upper right corner).

Table 1. Epoch deviations $\langle x\rangle$ and $\langle y\rangle$ in RA and Decl of VB 10 for the models with $\Psi=0$ and $\Psi \neq 0$ (Eq. (3)).

\begin{tabular}{rrrrrrrr}
\hline \hline \multicolumn{7}{c}{$\Psi=0$} & \multicolumn{1}{c}{$\Psi \neq 0$} \\
\hline $\begin{array}{c}\text { MJD } \\
55000+\end{array}$ & $\begin{array}{r}\text { RA } \\
\text { (mas) }\end{array}$ & $\begin{array}{r}\text { Dec } \\
\text { (mas) }\end{array}$ & $\begin{array}{r}\text { RA } \\
\text { (mas) }\end{array}$ & $\begin{array}{r}\text { Dec } \\
\text { (mas) }\end{array}$ & $\begin{array}{r}\sigma_{\mathrm{N}} \\
(\mathrm{mas})\end{array}$ & $\begin{array}{r}\sigma_{\text {sum }} \\
(\mathrm{mas})\end{array}$ & $\begin{array}{l}\sigma_{\text {fit }} \\
(\mathrm{mas})\end{array}$ \\
\hline 45.163 & -0.26 & -0.16 & -0.79 & 0.41 & 0.19 & 0.64 & 0.32 \\
82.032 & 0.20 & 0.19 & 0.15 & 0.35 & 0.10 & 0.18 & 0.16 \\
92.993 & -0.09 & -0.06 & -0.06 & 0.11 & 0.08 & 0.11 & 0.10 \\
97.014 & -0.00 & 0.18 & 0.03 & 0.08 & 0.07 & 0.11 & 0.09 \\
99.011 & -0.01 & -0.25 & 0.03 & -0.38 & 0.07 & 0.12 & 0.10 \\
\hline \multicolumn{7}{c}{ Short dataset, 8-25 Sept. 2009 } \\
\hline 82.032 & 0.07 & -0.09 & 0.33 & 0.43 & 0.11 & 0.19 & 0.16 \\
92.993 & -0.02 & -0.04 & -0.12 & -0.09 & 0.09 & 0.09 & 0.06 \\
97.014 & 0.03 & 0.07 & 0.02 & -0.00 & 0.07 & 0.08 & 0.05 \\
99.011 & -0.06 & -0.01 & -0.09 & -0.15 & 0.08 & 0.09 & 0.07 \\
\hline \multicolumn{7}{c}{}
\end{tabular}

Table 2. Probabilities $P\left(\chi^{2}\right), P(Q)$ and linear trends $Q$ (mas $\mathrm{yr}^{-1}$ ).

\begin{tabular}{rrrrrrrl}
\hline \hline Dataset & $\Psi$ & $\chi^{2}$ & d.o.f. & $P\left(\chi^{2}\right)$ & $Q_{\alpha}$ & $Q_{\delta}$ & $P(Q)$ \\
\hline full & 0 & 10.6 & 7 & 0.16 & 0.67 & -0.35 & 0.36 \\
full & $\neq 0$ & 18.0 & 7 & 0.02 & 4.14 & -5.38 & 0.02 \\
short & 0 & 1.9 & 6 & 0.93 & -2.06 & 2.47 & 0.21 \\
short & $\neq 0$ & 14.8 & 6 & 0.02 & -7.82 & -11.32 & $5 \times 10^{-4}$ \\
\hline
\end{tabular}

effective parameters for the short and the full datasets, respectively. The probabilities $P\left(\chi^{2}\right)$ corresponding to the $\chi^{2}$ values are given in Table 2 . For both the short and the full datasets, they are equally small at the $2 \%$ level if the model assumes $\Psi \neq 0$. Because the probability of this event is low, the large scatter of $\langle x\rangle$ and $\langle y\rangle$ is unlikely to be caused by random noise in the observations.

Secondly, we noticed that the observed residuals $\langle x\rangle$ and $\langle y\rangle$ show a linear trend instead of a random distribution. This can be caused by random errors in the observations, but also may indicate a wrong value of the signal $\Psi(t)$ subtracted in Eq. (3). In the latter case, we would expect that the residuals $\langle x\rangle$ and $\langle y\rangle$ show a linear dependence $-Q t$ opposite to $\Psi(t)$ (see Eq. (2)). 

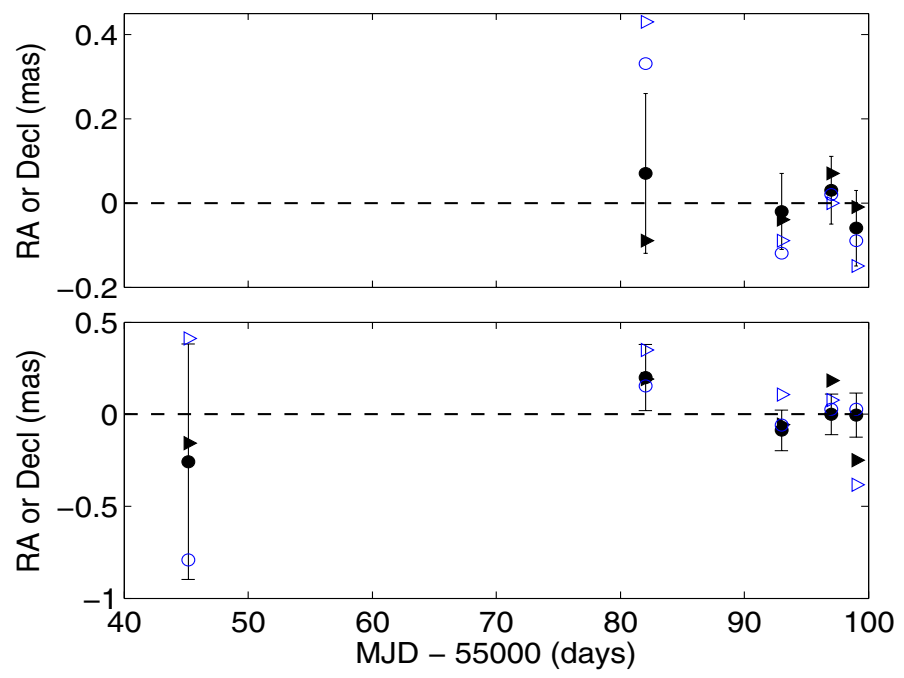

Fig. 6. Astrometric residuals in the scenario with planet (open blue symbols) and without planet (filled black symbols) for the short dataset (top panel) and the full dataset (bottom panel) as a function of time. Circles and triangles mark residuals in RA and Decl, respectively. For clarity, only one errorbar corresponding to $\sigma_{\text {sum }}$ is shown at each epoch, but the uncertainties for the four respective measurements are identical.

A strong negative trend is seen for the short dataset residuals computed with $\Psi \neq 0$ (Fig. 6), whereas they should be near zero to support the planetary hypothesis. In contrast, a much weaker trend is observed when assuming $\Psi=0$ (no planet). For instance, the measured values of $Q$ given in Table 2 for

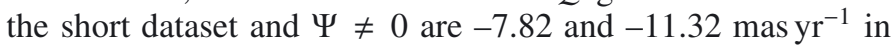
RA and Decl, respectively. If the null hypothesis $\Psi \neq 0$ is cor-

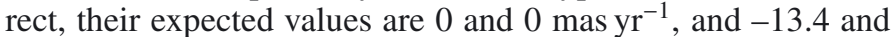
$-22.4 \mathrm{mas} \mathrm{yr}^{-1}$ if the hypothesis is wrong $(\Psi=0)$. In the last case, the measured $Q$ values are always lower than the values $Q$ (PS) induced by the orbital motion, because their magnitude is damped by correlations between $\Psi(t)$ and model parameters (cf. Sect. 3.8).

Because the $\chi^{2}$ criterion is not the most efficient one for characterizing linear signals, we developed another, more powerful approach to obtaining an alternative estimate of $P$. We considered $Q$ as quantities describing the dispersion of $\langle x\rangle$ and $\langle y\rangle$ better than $\chi^{2}$ and performed Monte Carlo simulations to see if the observed features can be explained by random noise in the observations. We simulated each data frame by a random Gaussian noise with root-mean-square of $\sigma_{1}$ and added components modelling the errors in $\mu^{\prime}, b$, and in the proper motion and parallax of VB 10. In addition, we included a $1 \mathrm{mas} \mathrm{yr}^{-1}$ error to account for the uncertainties in the zero point of proper motion (cf. Sect. 3.3).

After fitting the data with the model Eq. (3), we searched the residuals for linear trends in time and estimated their coefficients $Q^{*}$. The false-alarm probabilities $P(Q)$ that a random noise produces higher $Q^{*}$ values than the observed ones are given in Table 2 . In the case of the short dataset and $\Psi \neq 0$, we find that the observed linear trends can be explained by Gaussian noise with a probability of $P(Q)=5 \times 10^{-4}$, which does not support the existence of the planet. For the full dataset, we find $P(Q)=0.02$, which is not sufficient to draw definite conclusions. In spite of the longer timespan, hence seemingly better conditions for characterizing a planetary signal, the full dataset does not provide a better constraint because of the uncertainty in $\mu^{\prime}$, whose contribution increases with time. Besides, the planetary signal is substantially damped because of its strong correlation with the colour correction $\pi^{\prime}$ used in Eq. (3) as a free model parameter.

\section{Conclusions}

We conclude that the presence of the announced planet around VB 10 is not supported by astrometry. Even assuming half the planetary mass (i.e. 3.2 $M_{\mathrm{J}}$ ), simulations give a low false-alarm probability $P(Q)=0.023$, which still does not prove the existence of VB 10b. Our result obtained from astrometry alone agrees with the conclusion of Bean et al. (2010) and AngladaEscudé et al. (2010) based on RV data.

This study is the first application of the FORS2 camera in the search for exoplanets by means of optical astrometry. Because of the high astrometric precision of FORS2, the availability of external STEPS-based proper motion and parallax of VB 10, and the strong expected orbital signal, it was possible to verify the planetary companion hypothesis within the extremely short observation period of 17 days, which is unusual for astrometric works of this type. Successful use of STEPS data for reduction of FORS2 observations is only justified because we verified that the STEPS and FORS2 proper motion reference frames are con-

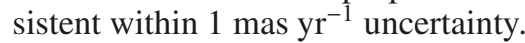

We have demonstrated a mean nominal precision of 0.09 mas per epoch of FORS2/VLT observation for data of reasonable quality, despite the problems caused by the uncertainty in the LADC position. This precision is sufficient for astrometric detection of planets around ultracool dwarfs.

Acknowledgements. We thank Dr. G. Anglada-Escudé whose comments have helped to improve the paper. P.F. and N.C.S. would like to acknowledge the support by the European Research Council/European Community under the FP7 through a Starting Grant, as well as the support from the Fundação para a Ciência e a Tecnologia (FCT), Portugal, in the form of a grant with reference PTDC/CTE-AST/098528/2008. NCS would further like to acknowledge the support from Fundação para a Ciência e a Tecnologia (FCT), Portugal, through a Ciência 2007 contract funded by FCT/MCTES (Portugal) and POPH/FSE (EC).

\section{References}

Anglada-Escudé, G., Shkolnik, E. L., Weinberger, A. J., et al. 2010, ApJ, 711, L24

Appenzeller, I., Fricke, K., Fürtig, W., et al. 1998, The Messenger, 94, 1 Avila, G., Rupprecht, G., \& Beckers, J. M. 1997, in SPIE, 2871

Bean, J. L., Seifahrt, A., Hartman, H., et al. 2010, ApJ, 711, L19

Lazorenko, P. F. 2006, A\&A, 449, 1271

Lazorenko, P. F., Mayor, M., Dominik, M., et al. 2007, A\&A, 471, 1057

Lazorenko, P. F., Mayor, M., Dominik, M., et al. 2009, A\&A, 505, 903

Pravdo, S. H., \& Shaklan, S. B. 2009, ApJ, 700, 623

Zapatero Osorio, M. R., Martín, E. L., del Burgo, C., et al. 2009, A\&A, 505, L5 